\title{
La Station apicole de Pita (Guinée française)
}

\author{
paT G. FORTIN
}

\section{VUE GENERALE}

L'abeille et ses productions représentent une richesse naturelle prodigieuse qui déborde le cadre de la Guinée et intéresse aus même titre I'A.O.F. et 1'A.E.F.

La Station apicole de Pita, si elle n'est pas située dans une contrée spécialement mellifère, n'en occupe pas moins géographiquement une position excellente lui permettant de rayonner sur la Guinée et même sur l'A.O.F.

Son climat d'altitude y est bon pour l'Européen.

Ses populations, ici très primitives, ont tout à acquérir de la science apicole qui n'en est même pas au stade de l'apiculture fixiste des indigènes nord-africains, mais encore en partie, comme pour diverses autres productions, au stade du produit de cueillette.

Les périodes de pénurie en matières sucrées, conséquences des conflits mondiaux, ont révelé les possibilités d'apport des colonies en miel et la Guinée $\mathrm{s}^{\prime}$ inscrivait en 1947 avec près de 5.000 tonnes.

Ce miel, extrait par des procédés très primitafs, se trouve, dès l'origne, souillé par le jus de larves, obtenu par pressage de tous les rayons et en fait un produit qu'aucun procédé mécanique ne saurait purifier.

Instable et même parfols toxique, ce produit a vu, avec le retour du sucre, se fermer devant lui tous les débouchés, privant ainsi le paysan noir d'une source de revenu considérable.

Il n'est, cependant, pas de cas d'abeille élaborant un miel impropre à lạ consommation.

\section{SA CREATION - SON PROGRAMME}

La création, à Pita, de la Station apicole fut décidée par M. le Gouverneur de la Guinée à la demande de M. le Chef du Service de l'Élevage.

Cette Station, sortie de la brousse, devait commencer à voir le jour par ses propres moyens en juillet 1950.
Les réalisatuons techniques se heurtent en ces pays à l'insuffisance d'artisans et ouvriers capables ainsi qu'à un équipement mécanique encore trop rudimentaire.

Le programme comprend :

\section{A. - Étudier l'abeille de Guinée.}

1. Son comportement dans le pays, selon les régions. Son cycle annuel en fonction des miellées successives. Les rendements en abeilles, miel, cire, pollen et propolis.

2. Sa valeur comme cirière. Sa capacité de conversion en cire des mauvais miels, avec, parallelement, sa capacité de reconditionnement de ces mauvais miels.

3. Étude, en liajson avec des Stations apicoles d'Europe, de son comportement hors du pays et de sa Iésistance à certaines affections microbiennes et parasitaires.

4. Étude de ses parasites spécifiques et éventuellement d'affections microbiennes si elles se révélaient un jour.

5. Etude de ses possibilités dans la production de biostimulant (gelée royale).

\section{B. - Étudier la flore mellifère de la Guinée.}

1. Inventaire. Classification. Répartition.

2. Calendrier floral avec indice mellifère complété par un tableau microphotographique des principaux pollens permettant la détermination rapide de l'origine des miels et leur classification.

3. Recherche de la flore responsable du goût spécifiquernent « guinéen 》.

4. Essais d'introduction d'essences mellifères réputées, en jardin d'essais, à la Station.

En laison avec les Services forestiers : extension d'essences de reboisement mellifères et étude de la valeur meilifère des essences de reboisement actuelles.

En liaison avec les Services agricoles et les S.I.P. : extension des plantations d'agrumes. 
C. - Dans l'immédiat, tirer partie de la richesse naturelle que représente "l'abeille " et ses productions : le miel, la cire, le pollen, la propolis.

1. L'abeille : par exportation sous forme de paquets d'abeilles, d'essaim, de colonies sur cadres.

2. Le miel : par la recherche d'un procédé de collecte et d'extraction, s'adaptant aux mœurs du pays et conservant au miel ses qualités d'origine.

3. La cire : par sa purification par les procédés classiques.

4. Le pollen : par la recherche d'un procédé d'extraction permettant d'en tirer un produit exportable propre au nourissement de l'abeille d'Europe.

5. La propolis : par simple collecte sous sa forme d'origine.

D. - Créer une apiculfure extensive aussi rudimentaire que possible, destinée à amener l'indigène à comprendre que le fait de ne pas détruire annuellement son CAPITAL représenté par l'ESSAIM 'ET SON COUVAIN, lui permettra d'obtenir des rendements quatre ou cinq fois supérieurs.

La création d'une telle apiculture requiert la conception d'une ruche "apiculturable " (1) à terre, résistante aux termites, facile à protéger contre les feux de brousse et d'une capacité en harmonie avec l'abeille et les possibilités mellifères.

\section{E. - Valonisation des productions de l'abeille avec les ressources locales.}

\section{Miel :}

a) confitures d'agrumes, confifures d'autres fruits, pain d'épice, nougrats, hydromel;

b) conversion des miels saisis ou impropres à la vente, en cire, par nourrissement;

c) utilisation des miels inférieurs dans l'alimentation du bétail et des volailles ainsi que dans la lutte antiacridienne en association avec H.C.H.:

d) distillation.

2. Cire. - Purification simple, confection de cire gaufrée, confection d'encaustiques et de mastics, sélection des cires extra-blanches de parfumerie.

3. Pollen. - Mise au point de son extraction et stabilisation du produit.

\section{F. - Propagande.}

Redonner confiance dans le miel par la recherche d'un prix de départ largement payant.

(1) Mot créé pour la circorzatance.
Multiplier les démonstrations d'extraction des ruches traditionnelles, au pied de l'arbre et en plein jour, pour faire valoir à l'indigène les inconvénients de sa ruche et le familiariser avec l'abeille et le matériel afin de lui faire dominer sa crainte.

Introduire la notion du respect du couvain et de l'utilité de récolter un essaim pour en peupler une ruche, plutôt que de s'en remettre à la Providence.

Tenter de faire naitre des vocations apicoles en montrant que, par son rapport, l'abeille peut justifier l'activité d'un homme avec le minimum de peine.

Faire comprendre l'unanité de la pratique des feux de brousse, destructrice de la source de cette inepuisable richesse, le NECTAR, et, partant, la rentabilité de la reprise par la brousse spontanée des sols sans vocation particulière.

Farre construire des ruches en paille genre "Bournat \#, diffuser la ruche en ciment genre «Djebah».

Installer par canton des ruchers rentables et exploitables en métayage à mi-fruit avec les S.I.P.

En liaison avec les sections manuelles des écoles et les coopératives scolaires : confection de ruches et ruchettes en paille et en bois, installation de petits ruchers scolaires, de ruches de démonstration, installation de ruchers dans les écoles d'agriculture, visites des écoles à la Station.

Ouverture d'une école d'apiculture à la Station, susceptible de recevoir " au pair » des candidats « volontaires 》 de toutes origines, même métropolitaines, donner une instruction de base accélérée au personnel de l'Élevage principalement en vue de la traite, former de jeunes apiculteurs pour la prise en métayage des ruchers de canton.

\section{SON ACQUIS}

\section{A. - Etade de l'abeille de Guinée.}

1. Race. - L'abeille de Guinée est du genre Apis, espèce Mellifica avec deux variétés Adansoni et Unicolor, en métissage constant, à prédominance d'Adansoni. Aucune autre espèce n'a été encore observée.

Taille, - Sa taille est nettement plus petite que celle d'Europe et d'Afrique du Nord. Elle bâtit en effet de 990 à 1.050 cellules au décimètre carré contre 800 à 850 pour l'Europe et l'Afncue du Nord. Les mâles sont également plus petits, alors que les reines semblent de taille normale.

Cemeure. - Elle adopte comme demeure n'importe quelle cavité naturelle ou artificielle : ligneuse (souches et troncs creux, caisses vides, paniers, meubles); de-pierre (anfractuosité de Iochers, vieilles canalisations, buses); de terre (termitières abandonnées), etc. Il lui arrive très 
fréquemrnent de bâtir en plein air, à l'intérieur d'un bâtiment. accrochant ses rayons à l'angle d'une porte ou d'une fenêtre, parfois même directernent sous la tôle ondulée: les rayons atteignent parfois des dimensions étonnantes : 1,50 mètre de hauteur.

Orientation des rayons. - Dans les ruches, les rayons sont le plus souvent disposés à « bâtisses froides ", c'est-à-dire orientés dans le sens de la plus grande dimension, alors qu'en Europe et en Afrique du Nord l'abeille bâtit plutôt à « bâtisses chaudes \%, c'est-à-dire perpendiculairement à cet axe.

Écartement des rayons. - L'examen de l'écartement des rayons d'axe en axe a donné de 33 a 40 millimètres avec une moyenne de 35 millimètres contre 37 à 38 millimètres en Europe. L'abeille de Guinée mise sur cadres à 37 millimètres d'écartement accepte cette dimension. Elle édifie relativement peu de cellules de mâles.

Les essaims. - Les colonles sont en général petites, un essairn naturel atteint rarement le kilogramme. Il a fallu les populations de 18 colonies pour constituer 12 paquets d'abeilles de 0,900 kilogramme.

L'extraction, dans un magasin de Boké, d'une colonie établie à l'air libre, dont les rayons atteignaient 1,50 mètre de haut, a donné 40 kilogrammes de miel pour une colonie n'atteignant pas 2 kilogrammes d'abeilles. Il va sans dire que la grappe n'occupait pas toute la surface des rayons dont la base était uniquement garnie de pollen. Les abeilles assuraient, cependant, une garde vigilante contre une pullulation de cancrelats; par contre, une quantité de petits coléoptères, Ethina tumidula, semblant vivre en commensaux, evoluaient sur les rayons sains être inquiétés.

Les essaims quoique petits font preuve d'une activité remarquable tant au point de vue sécrétion cirière que récolte de miel.

La miellée la plus importante ayant lieu en saison sèche, les abeilles butinent avec une intensité prodigieuse au lever du jour et à la tombée de la nuit, accusant un calme presque total au milieu de la journée.

Dans les Aindés, ceitaines ruches peuplées en janvier-février sont récoltées en avril-mai et, repeuplées à celte épocue, sont à nouveau récoltées fin août pour donner les miels dits de mais.

Dans l'ensemble, les abeilles sejournent moins d'un an dans la ruche avant d'être détruites, alors qu'en France un essaim enruché sur cire gaufrée et nourri-ne donne réellement que la- deuxième année.

L'essaimage s'étend a longueur d'année, avec son maximum d'intensité en mai-juin, provenant, d'une part, de l'essaimage des colonies sauvages et, d'autre part, des abeilles ayant réchappé au massacre de la récolte.

Les colonies élèvent quelques mâles en avril en prévision de cet essaimage et les refoulent des qu'ils ne sont plus d'aucune utilité.

Les essaims se laissent capturer assez facilement mais désertent, dans la moitié des cas, la demeure qu'on leur impose, quelle qu'en soit la nature, pour en adopter souvent une identique dans le voisinage. Cette désertion n'est pas toujours immédiate, elle peut avoir lieu plusieurs jours après enruchage alors qu'il y a déjà eu ébauche de cire et même ponte. La mise en cagette de la reine pour fixer l'essaim a donné des résultats, mais dans quelques cas, l'essaim a abandonné sa reine pour s'installer dans une ruche déjà peuplée où il est admis sans difficulté. Ces cas d'essaims fusionnant sont assez frequents, mais si l'abeille est admise à pénétrer, la reine, en général, reste à l'extérieur, "ernballée " dans une petite pelote d'abeille, puis disparait au bout de quelques jours.

Ce manque d'individualité de l'essaim a été surtout observé à la Station lors de la préparation des paquets d'abeilles à expédier sur la France : 14 ruches indigènes peuplées furent amenées de nuit et disposées côte à côte sur des tréteaux, I'effondrement des rayons était presque total, ceux-ci furent retirés le lendemain matin ; il se produisit alors un essaimage collectif sur les buissons voisins, pour donner en définitive trois masses d'abeilles contenant chacune plusieurs reines.

Cycle annuel de l'abeille. - Le cycle annuel de l'abeille, à Pita, s'établit de la façon suivante : l'observation en a été faite sur une colonie installée dans une fenetre entre vitres et volets. Cette colonie fut entjèrement dépouillée de tous ses rayons par des pllleurs d'abeilles dans le courant du mois de juin 1950 avec fort pourcentage de mortalité. L'essaim n'abandonnait pas sa demeure et abordant la saison des pluies dans de très mauvaises conditions. Il devait édifier en juillet-août 6 rayons dont le plus grand avait environ 4 décimètres carrés, puis restait stationnaire d'août à décembre avec, cependant, un couvain constant. Quoique accusant un très net ralentissement pendant l'hivernage, la colonie n'a jamais connu l'inaction totale, butinant même par temps de petite pluie. Dès le mois de janvier, cette colonie se mit à prospérer avec une extrême rapidité donnant en 4 coupes, de février à mai, I6 kilogrammes de miel. La colonie dut être enlevée à cette époque pour rendre la case habitable, une coupe ou deux étaient encore possibles.

L'extraction des ruches indigènes faite au mois 


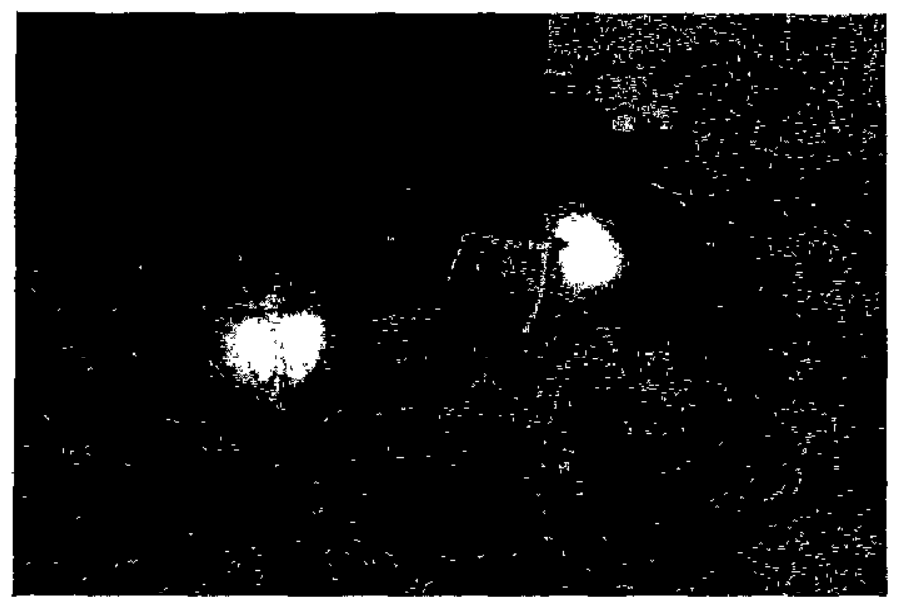

Figure 1.

Calebasses de miel prêtes à la livraison.

de mai 1950 a donné les quantités moyennes suivantes : miel 4.500 kilogrammes, cire environ 200 grammes, couvain et pollen 5 kilogrammes environ, pour un essaim de 700 à 800 grammes.

Des ruches extraites en arrière-saison juillet-août ont donné un miel très fin, jaune clair, mais avec un rendement dérisoire, 1 kilogramme par ruche.

Les faibles rendements des ruches sont imputables à leur capacité souvent trop petites et à la pratique de destruction de la colonie, qui n'est qu'annuelle dans les meilleures conditions, celle-ci pouvant avoir lieu à tout moment quand le besoin d'argent se fait sentir.

Le captif du Foulah ne détruit pas systématiquement l'essaim, lors de l'extraction de la ruche, celui-ci survit souvent, mais largement amputé.

Il sait extraire le miel proprement pour hi, par pressage à la main des rayons, mais, dès qu'il s'agit de le livrer au commerce, il ne manque pas de le frauder par adjonction d'eau, de jus de larve, en faisant un produit irtémédjablement soullé.

Le miel pressé, manipulé dans des calebasses dont il prend l'arnertume, est mis ensuite dans des touques à pétrole qui servent également à la traite de l'huile de palme. Ces touques servent de mesure dans les transactions.

Ruches indigènes. - Les ruches indigenes sont de types assez variés suivant les régions, tant par leur capacité que par leur confection.

La capacité varie d'une ving- taine de litres, dans les Aindés où les récoltes sont très rapprochées, à plus de 120 litres sur les plateaux Foulah à récolte annuelle.

La ruche la plus répandue est faite d'un cylindre central ayant de 15 à 40 centimètres de diamètre intérieur sur 0,75 à 1,50 mètre de long. Ce cylindre est fait d'un tronc d'arbre creusé à la gouje (spécialité des Labbos), d'écorce de deux arbres (tkiéwé, tkiapélégué) ou de bambous tressés dans les régions où la déforestation a détruit les ressources en arbres.

Le cylindre est ensuite recouvert entjèrement, ouvertures latérales cornprises, d'une première couche en feuille de bananier, ou de popoh selon les ressources du pays, puis de deux chapeaux de paille s'emboitant par les extrémités et dont les bords se recouvrant sont ligaturés sur le corps du cylindre par des lianes. Une fente passage d'abeilles de quelques centimètres est ménagée à la base ou latéralement.

On trouve encore en pays Foulah (région de Diaguissa) des nuches formées de deux coquilles en tronc creux se superposant. Ces ruches dépassent les 2 mètres de long et ont une capacité de plus de 100 litres; etant très lourdes, elles sont placées à la naissance des branches maítresses d'un arbre. La récolte se fait en enlevant la coquille supérieure à laquelle sont attachés les rayons. Dans la région de Ditinn, les ruches sont souvent faites d'un simple tronc creux obturé aux extrémités par un bouchon de paille.

En pays Cognaghi, les ruches sont faites en palle torsadée liée au Ronier, elles ressemblent au Bournat, mais au lieu d'être posées sur un plateau de bois à

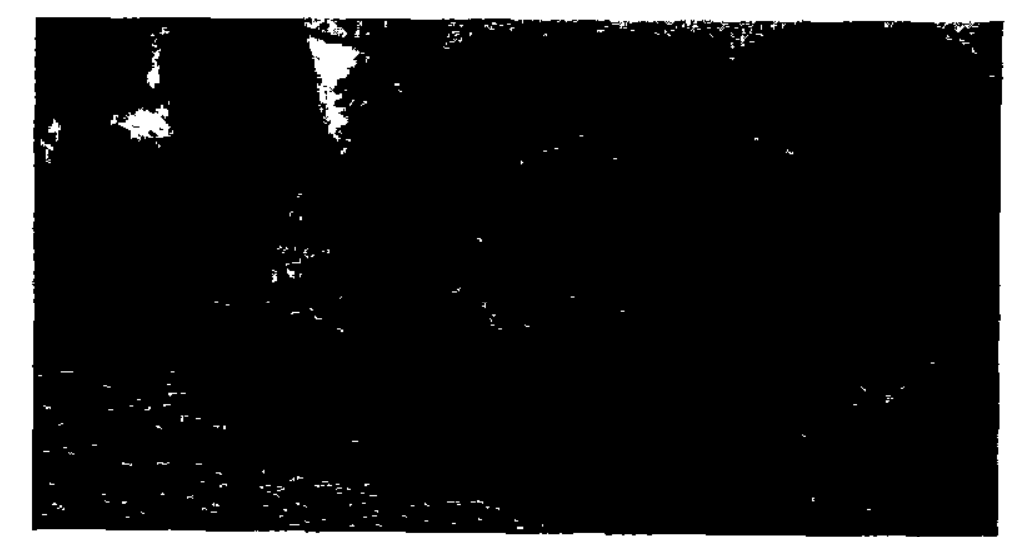

Figure 2.

Apiculfure en Guinée. - Dérails de ruches indrgènes 
terre, elles sont suspendues aux branches les plus hautes des plus grands arbres; la grande ouverture disposée horizontalement est fermée par une porte, également en paille torsadée, munie d'un trou passage d'abeilles en son centre.

Les fissures de toutes les ruches sont colmatées à l'aide d'un mélange d'argile et de bouse de vache.

2. Valeur cirière. - L'abenlle de Guinée est une remarquable cirière, qualitativement surtout, n'ayant pu être encore jugée sur la quantité. La cire est tellement pâle à l'origine que l'indigène croit la faire apprécier davantage en la colorant à l'hulle de palme. Purifiée, elle ne risque aucune concurrence sur aucun marché, car ici aucune abelle ne sécrète de cire brune. L'indigène la livre en boule sur les marchés après une première épuration.

Ira sécrétion cirière, essentiellement saisonnière et très courte en Europe, est ici à peu pres permanente, car elle est régie par deux facteurs : tempé-

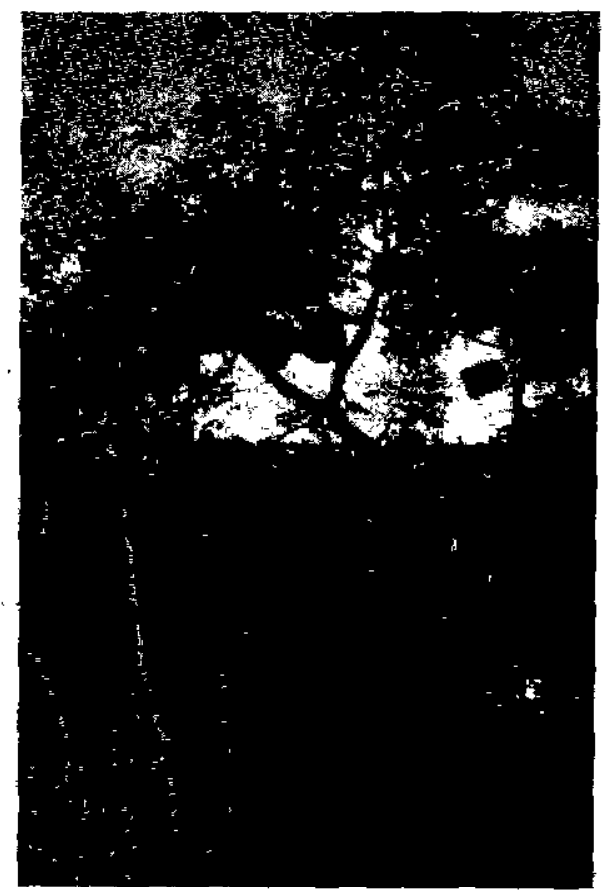

Figure 3.

Ruchetles en position de peuplement dans un nere. rature assez élevée et nectar constant. Elle accuse, cependant, un très net ralentissement pendant l'hivernage qui se situe de juin à octobre.

Le nourrissement est capable de maintenir cette sécrétion d'une manière constante, une trop courte experience a été faite à la Station utilisant des mauvais miels achetés à bas prix sur le marché. L'expérience sera poursuivie en période d'hivenage et il sera intéressant de noter exactement la capacité de conversion en cire de ces mauvais miels ainsi que la quantité et la valeur des miels reconditionnés par l'abeille. Même si l'on admet que 13 kilogrammes de miel sont nécessaires à l'élaboration d'un kilogramme de cire, l'opération reste rentable, le miel valant de 8 à 10 francs le kilogramme et la cire brute en boule des indigènes 150 francs le kilogramme $(13 \times 10=130$ francs $)$.

3. Dès que la ligne aérienne militaire de Labé séra doublée d'une ligne civile, il sera possible d'étudier en liaison avec des Stations apicoles d'Europe, le comportement de l'abeille de Guinée hors de son pays.

II $\mathrm{y}$ a lieu de noter ici que la colonie d'abeilles de Guinée, ramenée en 1949 par le Dr Matis et installée à Pasteur de Tunis s'est parfaitement acclimatée.

II sera intéressant d'étudier sa résistance possible à certaines affections microbiennes et parasitaires telle l'acariose.

Pour lutter contre cette maladie parasitaire, on a tenté de sélectionner une race à spiracles thoraciques plus étroits unterdisant l'accès à l'acarien. Ces essais faits en partant d'une abeille édifiant de 750 à 850 cellules au décimètre carré semblent avoir donné des résultats négatifs, alors qu'ils auraient pu être positifs en partant d'une race édufiant à plus de 1.000 cellules au décimètre carré. 


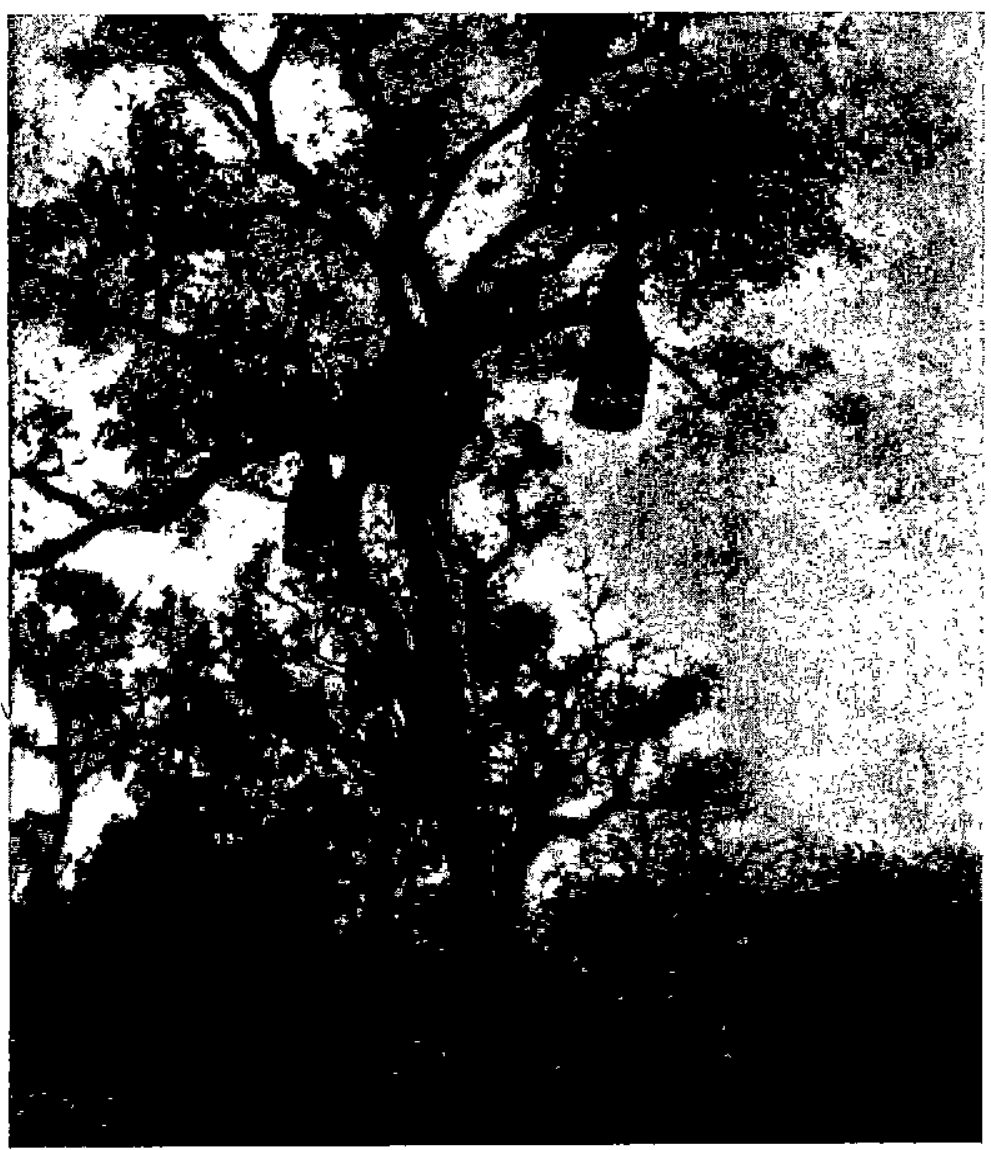

Figure 5 .

Ruches en paille rerticales

Il serait, en outre, intéressant de connaitre le comportement d'Ethina tumidula qui infeste son essairns mais qui, d'après le Prof. Balachowski, aurait peu de chance de s'acclimater en Europe. Ceci pour le cas où l'abeille de Guinée serait exportée.

4. Si du point de vue microbien il n'a jamais été observé, ni sur l'abeille, ni sur les nombreux couvains manipulés, la moindre trace de maladie, il n'en est pas moins vrai que les miels soumis à l'examen à l'Institut Pasteur de Paris et au Laboratoire apicole de Nice ont révélé la présence du Bacillus Alvej. Il y aurait lieu de tenir compte de ce facteur important dans les exportations d'abeilles et des produits de la ruche a destination de l'apiculture métropolitaine. Les parasites et déprédateurs sont ici extrêmement nombreux dans des ruches impossibles à défendre et sont peut-être la cause de l'instabilité de l'abeille.

Aucun de ces parasites n'a encore été étudié à la Station. Ceux simplement observés sont : Athina tumidula, parasite spécifique des pays tropicaux, Cétonia Cardui, connu en Europe et en Afrique du
Nord, il a ici des mours différentes, vivant d'ure manière permanente dans la ruche comme Fthina et se déplaçant comme lui avec l'essaim. Il est fréquent de récolter un essaim naturel sur une branche avec son escorte d'Ethina et de Cetonia.

Braula coeca, très connu en Europe et en Afnque du Nord, est également fréquent en Guinée; une reine capturée à Boké n'en comptait pas moins de 18 sur son corselet. Galeria melonella ou fausse teigne semble moins active qu'en Afrique du Nord.

On peut encore noter conme insectes : le sphinx, les blattes et cancrelats ainsi que de nombreuses variétés de fourmis dont une petite noire semble spécialisée dans l'attaque des jeunes colonies, qui désertent, abandonnant leur couvain aux pilleuses.

Le katori (en Foulah), appelé vulgairement margouyat, fait une très grosse consommation d'abeilles ainsi qu'un oiseau, le tnguérou (en Foulah). Une quantité d'autres oiseaux dont plusieurs espèces de guépier apparaissent par périodes.

Certains rats, les iguanes, et les singes causent parfois des dégâts appréciables.

5. De tout temps, l'indigène a su mettre à profit les vertus régénératrices du couvain, sans se préoccuper de la présentation, et l'on vient de découvrir que la gelée royale avait un pouvorr stimulant extrêmement puissant. Il est en effet frappant de constater que partant d'un ceuf fécondé la larve nourrie à la bouillie larvaire donnera, en un cycle de vingt et un jours, une femelle imparfaite l' " abeille », alors que le même couf dont la larve sera nourrie à la bouillie royale évoluera en quinze jours pour donner l'insecte parfait la " reine ».

Les possibilités de collecte de cette gelée, cotée quatre millions et demi le kilogramme, sont très farbles en Europe et peuvent être ici très importantes.

\section{B. - Etude de la tlore de Guince.}

1. Elle se présente sous un aspect assez particulier qui semble être la résultante de la pratique des feux 
de brousse opérant une sélection à rebours. Les sols dénudés par le feu sont envahis par une flore de graminées dont quelques-unes, bien rares, sont pollinifères, peu ou pas de flore spontanée; Boerrarja radiata, herbe adventice envahissante d'origine américaine considérée comme néfaste, est très butinée. La flore mellifère est presque entièrement composée d'arbres, d'arbrisseaux et de lianes.

Aucune etude systématique $\mathrm{r}^{\prime} \mathrm{a}$ encore été faite. Un herbier a été commencé à la Station.

2. Le calendrier floral et l'étude des pollens ne poursont être faits qu'après étude de la flore.

3. L'étude de 1. néen » sera entreprise le plus tôt possible pour la situer dans le temps.

4. Des essais d'introduction d'essences mellifères réputées ont été ébauchés à la Station et seront poursuivis. Partant de graines, les résultats sont longs.

Essences introduites et leurs résultats :

- romarin : bonne réussite;

- thym (vulgaris) : résultat médiocre, la variété (capitatus) devra donner des résultats;

- lavande et bruyere : pas de germination;

- pyrèthre, basilic, bourrache : très bonne végétation:

— sainfoin d'Espagne, lupin, soja : médiocre;

- hysope, sauge, réséda, giroflée, mélisse : très médiocre:

- luzeme arborescente : bonne germination;

- sophora, caroubier, melia, Schinus molle : bonnes germination et végétation;

- plaqueminier, févier : bonne germination, pas de végétation:

- tilleul, pittosporum, cognassier, sorbier, ronce, crataegus, laurus, eleaginus, celts : pas de germination.

Essences déjà introduites :

- bigaradier, néflier du Iapon, opuntia, fcus : très bonne végétation;

- zinnia, balsamine, ceillet d'Indes, pourpier, lantana, fraisier : se reproduisent à l'élat spontané;

- le mûrier qui se bouture avec une étonnante facilité et croit avẹc une très grande rapidité peut donner une contribution utile par son fruit.

Une liste d'essences de reboisement mixte, bois de feu, alimentation humaine ou animale et mellifères a été adressée au Service forestier, en vue d'essais.

Le Cassia siamea, actuellement très utilisé en reboisement, sera étudié au point de vue mellifère.

Le pin, comme tous les conifères, n'est d'aucun intérêt apicole. Générateur de miellat, il est néfaste.

Les agrumes, qui se reproduisent ici à l'état spontané, sont dans leur pays d'élection et pourraient même être envisagés comme essence de reboisement payante par la fleur, le fruit, la feuille. C'est, en outre, une plante mellifère de premier ordre. Ia question sera étudiee avec les Services agricoles et les S.I.P.

\section{C. - Dans l'immédiat, tirer partie de la richesse que représente l'abeille et ses productions.}

1. L'abeille. - La proposition du $D^{r}$ Mathis, à savoir : "Expédition de ruches indigènes entières, par bananiers, en chambres légèrement réfrigórées " s'est avérée immédiatement irréalisable, car l'effondrement des rayons, malgré les précautions prises, a été presque de règle et ceci est normal pour des brèches de cire nouvelle puisqu'elle n'atteint jamais un an.

A la faveur de cet efiondrement, un parasite spécifique de l'abeille tropicale Ethina tumidula qui vit habituellement en commensal sars préjudice apparent développe une ponte prodigieuse, transformant, dans les quarante-huit heures, le contenu d'une ruche en we masse grouillante d'asticots. L'essaim déserte en général la ruche dans les vingtquatre heures de son transfert,

Ira Station s'est alors orientée vers l'expédition du paquet d'abeilles pour les raisons suivantes:

a) en raison de sa grosse demande sur le marché métropolitain;

b) en raison de son anonymat; en effet, l'abeille de Guinée étant un métis constant entre l'abeille noire et jaune, son introduction en France, en colonies complètes, aurait pu justifier des critiques, alors que le paquet d'abeilles dépourvu de reine et de mâles n'est qu'un apport de butineuses en début de miellée, qui ne laisse plus aucune trace en fin de miellée si ce n'est une rentrée de miel appréciable.

A la suite d'une annonce parue dans la Revue Irançaise d'Apiculture, d'avril 1951, il fut demandé à la Station près de 2.000 paquets d'abeilles ou essaims. Une premère tentative d'expédition fut faite le 6 mai 1951 portant sur 12 paquets d'abeilles de 900 grammes mis en canssettes spéciales grillagées.

$A$ cet effet, 14 ruches indigènes furent achetées en brousse et extraites au Rucher du Centre presque simultanément dans l'après-midi du 5 mai. Les populations de ces 14 colonies s'amalgamaient en 3 groupes sur des buissons voisins. I fut puisé dans ces masses pour remplir les caissettes d'expédition. La grille destnée à retenir les reines et les mâles n'ayant pas fonctionne, les caissettes furent remplies 
de tout venant, y compris le parasite Ethina tumidula qui, d'ailleurs, en raison de sa taille exiguë, n'aurait pu être éliminé par filtration.

Étant donné la faiblesse des populations des ruches indigènes (pourtant bâties entièrement pour la plupart), il fallut prélever quatre ruches de la Station pour compléter les 12 paquets d'abeilles.

Cette première expédition ne devait pas être couronnée de succès. Il faut en voir les raisons :

- dans les mauvaises conditions de transport, mauvals état de la route provoquant la destnuction permanente de la "grappe 》, seule forme sous laquelle l'abeille peut vivre; il faut noter également des circonstances météorologiques defavorables;

- dans la longueur du transport (effectué uniquement par route) : en effet, ces colonies ont subi un premier transport, de la région du Kinkon au Centre apicole, dans la nuit du 4 , mise en ruchettes lo 5 , elles prenaient la route le 6 pour ne pouvoir être embarquées à Conakry que le 10 (l'embarquement ne put avoir lieu à cause de la trop forte mortalité).

Ces délais de transport sont beaucoup trop longs, mais il y a lieu cependant de noter qu'un essaim témoin a été conservé au laboratoire pendant huit jours avec une mortalité normale.

Un deuxième essai d'expédition d'abeilles a été fait le 3 juin portant sur deux colonies avec reines, l'époque étant trop tardive pour le paquet d'abeilles. Ce deuxième envoi préparé le 2 juin empruntait la route jusqu'à Mamou, puis le fer jusqu'à Conakry et était encore en état de prendre l'avion du 6.

A leur arrivée à la Station apicole de Bures-surYvette, l'un des deux essaims était mort et l'autre réduit à une poignée d'abeilles. Ce dernier désertait après avoir commencé un petit élevage de couvain.

Ces premières tentatives d'expédition avaient pour but de connaitre le comportement de l'abeille de Guinée en Métropole. Il semble à première vue, en raison de son comportement ici, que cela soit parfaitement possible.

L'expédition par Mamou-Conakry réalisait les plus mauvaises conditions de transport, de temps et de prix. En effet, un prix de transport avion ConakryParis de 1.600 franes C.F.A. équivaut au prox de vente en France. Si l'expédition de l'abeille de Guinée devait se réaliser, elle ne pourrait se concevoir que par avion cargo complet emportant dans les 650 colonies, chargées sur le terrain d'aviation le plus proche de Pita et pouvant effectuer le transport dans les quarante-huit heures maximum. Mars ceci suppose une organisation à l'arrivée et au départ.
2. Le miel. - La campagne de miel de 1952 a prouvé que l'indigène est disposé à livrer en quantité son miel en brèche en provenance, soit de ses niches, soit de colonies sauvages.

Le processus employé a été le suivant:

Des postes d'achat ont été installés en brousse, dans des cases, sous la direction d'infirmiers vétérinaires et stagiaires de la Station, avec mission de réceptionner le miel en brèche, livré par l'indigène en bassines émallées, en opérer le tri pour éliminer toutes traces de couvain et séparer les brèches de cire blanche et celles de cire brune, peser et payer sur-le-champ un prix allant du simple au double selon qualité. Les brèches sont alors mises en tonnelets à ouverture totale et expédiées sur le centre d'extraction-maturation.

Au centre d'extraction-maturation, les brèches sont émiettées à la main sur des couloirs à opercules, puis longuement égouttées.

Le miel soutiré des couloirs à opercules est mis en tonnelets maturateurs, une décantation de quinze jours a suffi pour le débarrasser des impuretés, la maturation physique ne présentait aucun intérêt, le miel accusant 1,42 à 1,43 de densité à la sortie du couloir à opercules, soit un taux voisin de $17 \%$ d'humidité.

Le miel soutiré des maturateurs est mis, le plus tôt possible, en tonnelets d'expédition de 165 ou 315 kilogrammes soigneusement fermés pour empêcher l'absorbtion d'humidité.

Les marcs de cire sont passés au pressoir type vigneron pour en extraire le miel de presse de qualité inférieure réservée au marché local. Enfin, ces marcs pressés sont envoyés à la fonte à la chaudière.

3. La cire. - Elle donne par les procédés de fontes classiques à la chaudière et au cérificateur solaire un produit de tout premer choix justement apprécié sur les marchés. Une difficulté surgit lorsqu'il s'agit de cire livrée par l'indıgène qui lui fait subir une première épuration assez grossière, mais, en contrepartie, la fraude trop souvent avec des pierres, du sable, de la ceridre, du sel, de la cire végétale et, étant souvent très pâle, il éprouve le besoin de la colorer à l'huile de palme.

4. Le pollen. - I est détruit en quantité considérable en pure perte, sa récupération mérite une étude.

5. La propolis. - Elle pourra être récoltée si la demande en est faite.

\section{D. - Créer une apiculture extensive rudi- mentaire.}

Les méthodes généralisées de récolte qui se pratiquent en Guinée, tant sur la ruche que sur la 
colonie sauvage, ne méritent, en aucun cas, l'appellaton d' « apiculture 》, puisque le résultat est la destruction de la colonie, c'est de l' « apidestruction \&. Il faut une nature prodigieusement généreuse pour permettre à l'abeille de survivre et prospérer avec le traitement qu'elle subit, auquel s'ajoute l'incendie annuel de la brousse qui détruit en quantité non chiffrable les colonies sauvages ainsi que Ia flore.

La ruche de Guinée (qui ne contribue que pour une part dans l'apport total du miel mis sur le marché, le surplus provenant de la clieillette des colonies sauvages) n'est pas " apiculturable» pour les raisons suivantes:

1. Elle est conçue pour être détruite et refaite chaque année, car elle ne comporte pour tout orifice qu'un trou passage d'abeilles de quelques centimètres disposé un peu au hasard.

Certaines ruches, cependant, telle la Cognaghi et quelques ruches de la région de Ditinn ont une porte, mais elles sont soumises à la mêrne destruction.

2. Presque toujours fixée dans les branches hautes j'un arbre, il faut l'amener à terre pour la décoriquer et l'extraire.

Les raisons invoquées pour justifier cette apiculture lérienne sont multuples. La routine, le -préjugé, la iuperstition et la crainte y ont une part préponlérante. Deux raisons méntent d'être retenues, qui ont en réalité deux fléaux : le " termite " et le ( feu $)$.

Aucun élément cellulosique mort ne peut être zaintenu au sol ni même à faible hauteur sans subir 'attaque du termite. Le feu de brousse détruit tout, ouvent même jusqu's'aux cases.

Ici, la nature donne un démenti à l'homme; en fiet, nombre de colonies sauvages élisent domicile ans des termitières abandonnees, des souches reuses, des trous de roches, prouvant l'inutilité e cette apiculture aérienne.

3. Par la nature des matériauxx employés, cette tche ne saurait tenir au-delà d'un hivernage; de là, pratique qui consiste à placer les ruches dans les bres à mi-saison sèche (janvier-février après les ux de brousse) pour récolter en avril-mai dans $s$ « Aindés $》(1)$ et en mai-juin pour récolter Innée suivante en avril-rnai dans les « Foutahs » (2).

(1) Vallée fertile.

(2) Plateau.

Ruches " apiculturables ». - Tenant compte de ces données, la Station a été amenée à concevoir deux types de ruches "apiculturables » à terre, I'une, du type horizontal, s'apparente à la «Djebah» des indigènes nord-africains, l'autre, du type vertical, dérive de la ruche à calotte de France.

Ruche du type horizontal. - Elle est faite d'une buse en ciment à embouts femelle-femelle de 30 centimètres de diamètre sur 1 mètre de long, soit 70 litres de capacité, disposée horizontalement et fermée à chaque extrémité par une porte ronde en bois ou en paille percée en son centre d'un trou passage d'abeilles.

A l'instar des ruchers arabes, ces ruches peuvent être groupées par dizannes et même par centaines selon la richesse mellifère de la région.

Le miel toujours situé périphériquement au couvain se trouve aux extrémités de la buse et est aisément extrait par coupe des rayons après un léger enfurnage.

Ruche du type vertical. - Elle est faite d'un " corps de ruche " en demi-buse de 40 centimètres de diamètre sur 50 centimètres de hauteur, soit 60 litres de capacité, reposant sur une dalle ronde en ciment formant " plateau 》, une ouvèrture passage d'abeilles de 20 centimètres sur 8 à 10 millimètres de hauteur est ménagée entre corps de ruche et plateau.

Le corps de ruche est obturé à la partie supérieure par une petite dalle en ciment percée en son centre d'un trou de 10 centimètres, une calebasse de 10 litres environ formant " calotte 》 recouvre la ruche. Le couvain occupe le corps de ruche qui n'est jamais touché et le miel est accumulé dans la calebasse qui est retirée pleine et remplacée par une vide aussi souvent que la miellée le permet. 


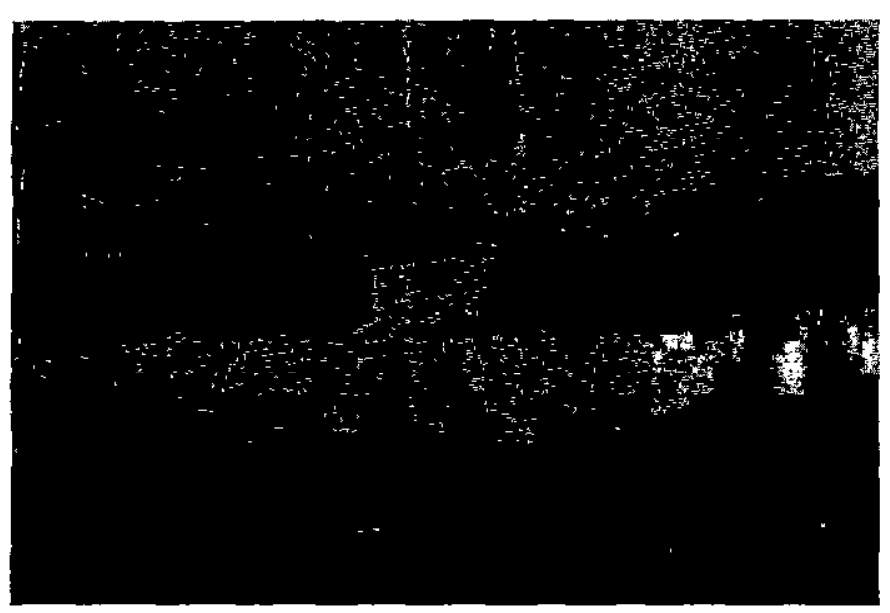

Figure 7.

Rucher de J00 ruches du trpe vertical. (miel et cire en brousse valant respectivernent 10 francs et 150 francs).

Inconvérients de ces ruches. - Ces ruches en ciment neuves ne sont pas attractives pour l'abeille comme le sont les riches traditionnelles indéfiniment récoltées et repeuplées, et il ne viendrait jamais à l'idée de l'indigène (sous peine de s'exposer à la risée générale) de capturer un essaim pour l'enrucher comme ceci se pratique en Europe et en Afrique du Nord.

Il est incontestable que l'abeille de Guinée n'a pas la docilité de l'abeille d'Europe. L'enruchement d'essaum en caissettes de bois n'a pas donné $50 \%$ de réussite et moins encore en ruches en, ciment. L'abeille semble vouloir choisir sa demeure. Par contre, le

Avantages de ces ruches. - Ces ruches « apiculturables » pourront :

- donner naissance à une apiculture rudimentaire, à la portée des populations primitives du pays;

- faire naître par groupage en rucher, la vacation et le métier d'apiculteur;

- détruire le préjugé de l'abeille qui doit changer annuellement de demeure;

- introduire la notion du " capital » abeillecouvain à respecter et de l" " intérêt » miel-cire à récolter:

- obtenir des rendements quatre à cinq fois supérieurs et donner des brèches propres, de dimensions susceptibles d'être désoperculées et passées à l'extracteur;

- permettre, par des récoltes successives, une sélection des crues en fonction des diverses floraisons et, partant, d'éliminer les miels responsables du goût spécifiquement guinéen, au mème titre que sont éliminés les miels de bruyère et d'eucalyptus en Afrique du Nord.

Ia calotte peut être remplacée par une " hausse " à cadres permettant au débutant toutes les fausses manceuvre sans risquer de compromettre la vie de la colonie, ouvrant ainsi la voie à une apiculture progressiste. Elle pourra encore guider les époques de récolte et jouer un rôle appréciable dans le reconditionnement des mauvais miels par l'abeille. Elle ne devra, toutefois, rester que sur le plan expérimental tant que le miel n'atteindra en valeur que le 1/15 de la valeur de la cire

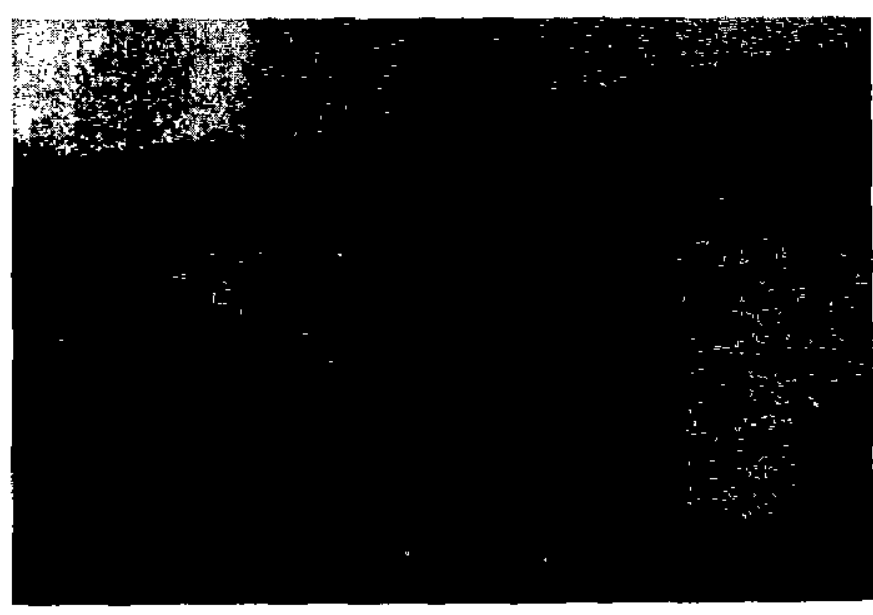

Figure 8.

Hausses a cadres langstroth sur ruches verticales. 
On peut admettre qu'une ruche en ciment peuplée l'est définitivernent, car si, même par suite d'une fausse manceuvre, la colonie arrivait à périr la ruche serait immédratement réoccupée.

Dans l'instauration d'une apiculture en ce pays, il y a lieu de noter ici un écueil sérieux. Il est, en effet, de pratique courante de consommer le couvain au même tstre qu'un gâteau; enfants et vieillards en font une grosse consommation, et jamais un vieux maitre Foulah ne reprochera à son captif de lui avoir récolté une nuche sans miel pourvu qu'il $y$ ait un abondant couvain. Grâce à lui, ił pourra, malgré l'âge, faire face aux exigences du harem.

Si dans la ruche du type horizontal, la tentation reste grande d'empiéter sur le couvain, celle-ci devient impossible dans la ruche du type vertical. Par contre, un rucher de 100 ruches horizontales ne demandera qu'un espace de quelque 50 mètres carrés facile à désherber contre le feu et clore contre les animaux et les maraudeurs, alors que pour un rucher vertical équivalent, il faudra dix fois plus d'espace.

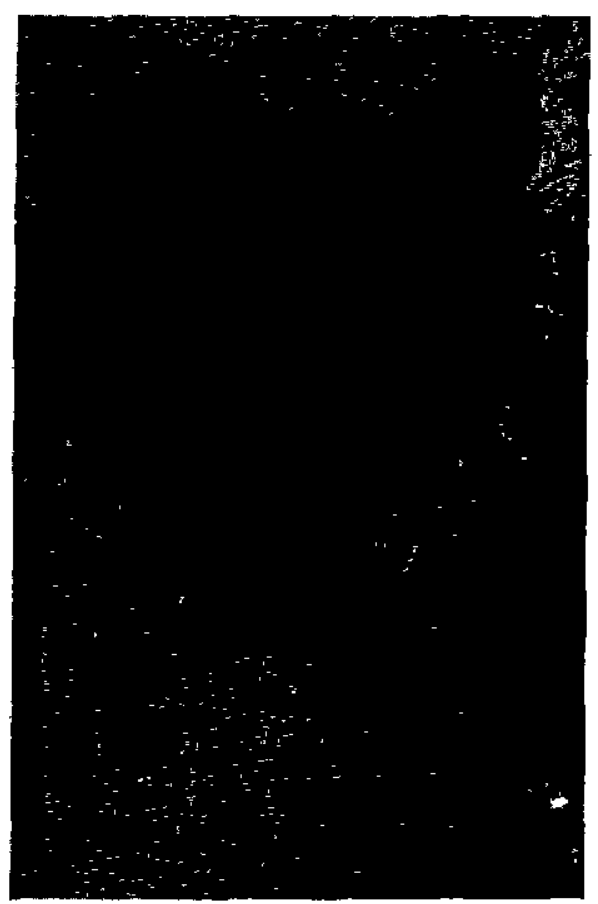

Figure 9.

Essaim prenant possession d'une ruche rerticale.

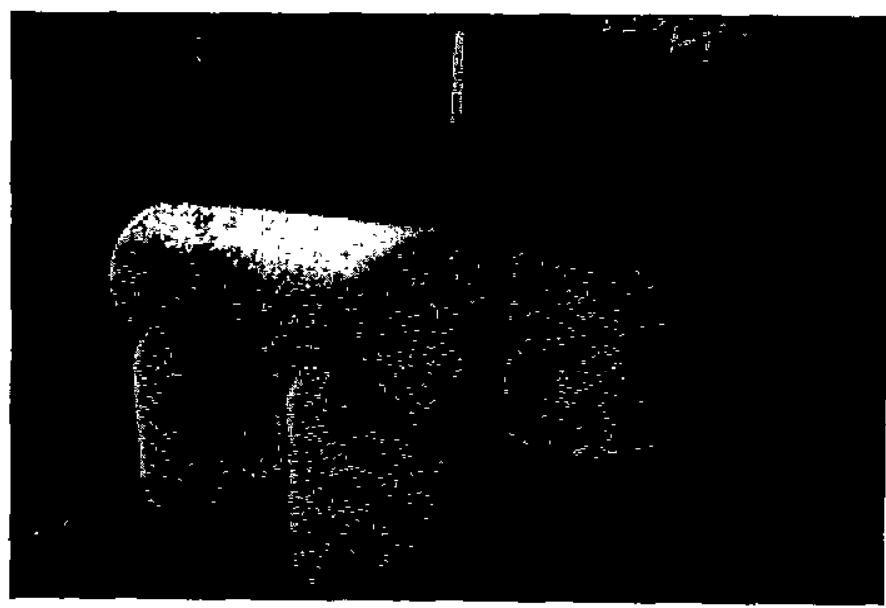

Figure 10.

Ruche du type horizontal genre " djebah v. Ruche du type vertical gense ruche d calotte.

Un rucher de 100 ruches horizontales ainsi qu'un rucher de 100 ruches verticales ont été installés à la Station de Pita et sont en cours de peuplement, l'abeille a parfatement adopté le ciment.

Un nucher de 100 ruches a été installé à la fermeélevage de Ditinn, 6 autres ruchers sont en cours de montage dans les cercles de Pita, Dalaba et Labé.

Mobilisme. - L'implantation d'une apiculture mobiliste n'est pas à envisager dans l'immédiat pour les raisons suivantes :

1. L'expérience a prouvé que cette apiculture n'a pas réussi à s'implanter parmi les populations indigènes d'Afrique du Nord et du Congo belge.

2. Cette apiculture est non productive de cire alors que ce produt est à ce jour le plus rémunérateur.

3. Cette apiculture n'est pas rentable car elle exige une muse de capitaux importants à amortir dans un court délai.

4. Cette apiculture ne pouvant s'adresser qu'à une élite à éduquer, elle frusterait la masse des paysans noirs d'un revenu qu'elle est en droit de conserver et d'améliorer.

En outre, le mobilisme pratiqué en pays tropicaux même par l'Européen a eu des fortunes très diverses, plutôt mauvaises, par suite du manque de continuité.

\section{E. - Valorisation des productions de l'abeille avec les ressources locales.}

1. Le miel.

a) Confilute d'agrumes, pur fruit pur miel. Au prix de l'orange en saison, moins de 5 francs le 
kilogramme, on peut obtenir une excellente confiture à moins de 20 francs le kilogramme départ. Pommelo, cédrat, citron, bigarades donnent aussi de très bonnes confitures.

Certains miels forts en gout et en colleur donnent de très bons pains d'épice, l'essai en a été fait à la Station. Un nougat aux arachides ou genre smyrne pourra être étudié avec débouchés en pays arabes.

La confection d'hydromels secs en vinification classique, irréalisable en Europe en raison du prix du miel, devient ici payante : 30 kilogrammes de miel à 15 francs, soit 450 francs, donnent un hectolitre de vin à $12^{\circ}$. Cette industrie peut absorber des miels inférieurs et utiliser les pollens perdus.

b) La conversion en cire, par nourrissement, des miels saisis ou impropres à la vente ainsi que le reconditionnement de ces miels par l'abeille, sera étudiée.

c) L'utilisation des miels inférieurs dens l'alimentation du bétail et des volarlles est à envisager et pourrait aider à résorber les stocks de mauvais miels qui pèsent sur le marché, à condition qu'ils ne soient pas toxiques.

Dans la confection des appâts acricides, les miels de rebut pourraient remplacer la mólasse et H.C.H. l'arsenic, idéo suggérée par le Prof. Balachowsky.

d) La distillation devra étre envisagée à l'échelon colonie pour résorber les stocks de miels impropres qui pèsent sur le marché el jouer, le cas échéant, le rôle de régulateur. 30 kilogrammes de miels à $B$ francs $=240$ francs donnent $12^{\circ}$ d'alcool à l'hectolitre, soit : $240: 12=20$ francs le degré alcool-hectolitre, qui donne pour un alcool a $90^{\circ}$ : $20 \times 90=1.800$ francs, soit 18 francs le litro à multiplier par deux pour frais de conversion $=36$ francs le litre.

2. La cire. - Le problème de la cire n'existe pas; néanmoins, sa slmple conversion en cire gaufrée en fait un produit à environ 1.000 francs métropolitain le kilogramme qui sera fabriqué à la Station.

La confection d'encaustiques, de mastics, ainsi que la sélection des cires extra-blanches de parfumerie pourra être étudiée.

3. Le pollen. - La mise au point d'un procédé d'extraction mérite une étude. Le pollen si nécessaire à l'abeille d'Europe est actuellement remplacé par des farines diverses. Il fait, en outre, actuellement l'objet d'étude, ẹn alumentation humane.

\section{F. - Propagande.}

Recherche d'un prix. - La recherche d'un prix de départ largement payant pourra redonner confiance au paysan noir dans cette production et l'unciter à multiplier les ruches de toutes sortes, traditionnelles et améliorées.

Un prix en brousse de 8 a 10 francs C.F. $A_{1}$ le kilogramme s'appliquant à un miel extrait par les procédés traditionnels (donc inférieur) est à peine rémunérateur; s'adressant à un miel extrait proprement, ces prix peuvent être multipliés par deux, soit 15 à 20 francs C.F.A. le kilogramme.

En effet, la commercialisation en France d'une tonne et demle de miel tout venant en provenance des cercles de Dalaba et Labé, exirait prcprement à la Station, a permis d'obtenir des prix allant de 125 à 200 francs, laissant entre 40 francs, prix d'aphat, et 125 francs, prix de vente, une marge de 85 francs pour manipulation, emballage, transport, taxes et bénéfice.

Éducation de base. - Les multiples extractions de colonies indigènes faites en brousse au pied de l'arbre et en plein jour semblent avoir eu surtout un intérêt spectaculaire. Il n'en est pas moins vral qu'elles demontrent qu'avec un. equipement approprié, l'abeille n'est plus un animal redoutable et qu'il peut être manipulé en plein jour.

Ces dérnonstrations prendront toute leur valeur lorsqu'elles s'appliqueront a une ruche amélioré " apiculturable » où le couvain ne sera plus détruit, ce sera là le rôle des ruchers de canton.

Le rucher de canton pourra, en outre, faire naitre le métier d'apiculteur : en effet, un rucher de 100 rudhes peut dónner 2 tonnes de miel à 20 francs: ceci représente 40.000 francs, soit en métayage avec les S.I.P. un salaire annuel de 20.000 francs pour une peine à pou près nulle ne representant pas trois mois de travail effectif.

L'apiculteur expioitant un rucher se rendra peutêtre compte de l'intérêt que représente pour lui la brousse envronnante et cherchera-t-jl le moyen de la protéger contre l'incendie.

Il faudrait aussi arriver à faire comprendre aux collectivités que la reprige par la brousse spontanée des sols sans vocation particulière peut être une opérations payante; grấce à l'abeille, on pourrait arriver à cette fin par des ruchers collectifs de village.

Des ruches seront diffusées dans toutes les écoles et principalement des ruches vitrées. Des démonstrations devront être faites régulièrement, c'est à cet échelon que les vocations ont le plus de chance de se révéler.

Il devra etre fait appel aux sections manuelles des écoles pour la confection des rucheltes de peuple ment pour le compte de la Station et des S.I.P.

Les coopératives scolaires pourront mettre ces ruchettes en peuplement à proximité des concessions et les céder peuplées à la Station et aux S.I.P. 
Les écoles d'agriculture et les fermes-écoles devront avoir un rucher d'exploitation.

La Station a édifié un local de 120 mètres carrés destiné à accueillır des stagiaires et leur faire siuvre un cours d'apiculture.

In laboratoire de rucher équipé en matériel modeme ainsi qu'un laboratoire pour le traitement de la cire (en voie d'achèvement) également équipé en matériel moderne ont été édifiés par la Station.

Le rucher de la Station comporte, en dehors des ruches en ciment, 20 ruches Langstroth à cadres peuplées. Un petit nucher couvert et vitré permettant de suivre l'évolution de l'abeille sans manipulation sera incessanment édifié.

La Station, si elle y est autorisée, pourra début 1953 recevoir " au pair " des candidats "volontaires" de toutes origines, principalement en vue de former des métayers pour les ruchers de canton. Une instruction accélérée sera donnée au personnel de l'Élevage principalement en vue de la traite du miel.

Une salie de l'école sera réservée comme salle de lecture et de démonstration à la disposition des élèves et de ceux que la question apicole intẻresse avec a leur disposition livres et revues concernant l'apiculture, l'élevage, la sériciculture et l'aviculture.

İn jardin botanique est en cours, avec détermination des essences mellifères introduites, la détermination des essences locales sera poursuivie.

Enfir, la Station compte une plantation séricicole de 5.000 mûriers, ainsi qu'une plantation d'agnumes d'un millier de plants.

\section{CRITIQUES ET SUGGESTIONS}

En envisageant le problème du miel de Guinée, problème commun à tout le continent noir, uniquement sous l'angle de la Guinée, on arriverait à brève échéance à un tonnage déja connu de 5.000 tonnes qui, commercialisé à 120 francs métropolitains (prix du sucre en France), donnerait un volume d'affaires de 600 millions laissant au paysan noir net en brousse. a 20 francs le kilogramme, une rentrée de 100 millions C.F.A. auxquels s'ajoutent la cire, et les autres productions quand elles seront valorisées.

Pour résoudre ce problène, il ne saurait être question d'une entreprise fonctionnarisée. Il appartient normalement au commerce de reconsidérer cette richesse dans le cadre des méthodes propres à onserver au miel sa pureté d'origine.

Le commerce, dans son organisation actuelle, est-il apte à drainer ce produit dès l'origine?

Le désir exprimé par la Charnbre de Commerce de Conakry dans son procès-verbal du 4 juin 1952 d'orienter les recherches vers la production de miel de bouche alors que le miel industriel trouve ces débouchés immédiats ne parait pas raisonnable.
II serait insensé de vouloir réformer la flore de Guinée pour éliminer le parfum guinéen qui, d'ailleurs, n'a rien de redhubitoire. La sélection des crus demandera du temps. Ir'extension des plantations d'agrumes permettra d'obtenir des miels comparables à ceux de Californie.

La position du commerce s'explique fort bien : un stock considérable de mauvais miel pèse encore sur le marché, stock qui ne pourra être résorbé que par la distillation.

Si le commerce ne désire pas reprendre cette activité, et que l'initiative privée ne se fasse jour. il y aura lieu d'envisager la formule "coopérative » qui groupera à l'origine un certain nombre de sociétés indigènes de prévoyance.

Cette formule permettant d'atteindre l'apiculteur en brousse aura la souplesse nécessaire pour la mise en valeur de cette énorme richesse dont le miel n'est qu'un élément. Agissant en coopératve de transformation, elle pourra valoriser au maximum les diverses productions de la ruche pernettant ainsi d'abaisser dans une large mesure le prix de revient du miel.

La prospection du marché métropolitain a prouvé qu'un miel de Guinée extrait et emballé correctement pouvait trouver un débouché industriel important et concurrencer les miels de Cuba et d'Argentine. Mais il faut pour cela arriver à une stabilité dans la qualité et une continuité dans l'alimentation de ce marché.

L'industriel en général s'est désintéressé du miel au bénéfice du glucose et du sucre interverti, d'abord par les bas prix de ces produits, ensuite parce qu'aucune obligation légale ne lui est faite d'utiliser du miel dans ses fabrications (pain d'épice et nougat notamment), enfin parce que le marché ne connait aucune stabilité de prix du fait des aléas des récoltes métropolitaines et des admissions capricieuses des miels extérieurs.

Pour reprendre place sur le marché industriel, il est nécessaire de fournir un produit de qualité au plus bas prix possible. La prix de 125 francs obtenu a Paris est certainement actuellement un prix plafond, c'est un prix qui, en tout cas, est largement rémunérateur pour la colonie.

Le matériel apicole que l'on trouve sur le marché et qui est prévi pour une apiculture classique a cadres répond imparfaitement aux besoins du traifement du miel de Guinée dont le point de départ est la brèche. Son rendement en est en général trop faible.

L'émiettage des brèches est nécessaire, car elles sont de trop petites dimensions pour être désoperculées et si elles étaient soumises à la pression elles libéreraient trop de pollen. Cet émiettage peut être réalisé mécaniquement par une machine 
très simple. Ces brèches émiettées sont ensuite essorées dans une essoreuse classique à grand rendement dont la cage devra avoir une maille étudiée. Ce matériel peut équiper un véhicule fermé se rendant sur les lieux de collegte des brèches. II se peut, en années à pluies précoces ou en région à hygrométrie élevée, que le miel (très hygrométrique) ne puisse mûrir, c'est-à-dire évaporer son excès d'eau et, de ce fait, ne pouvoir se conserver. Le chauffage étant interdit, sa maturation rapide peut être obtenue sous vide. On a en toute circonstance intérêt à abaisser le taux d'humidité du miel au bénéfice de sa conservation.

\section{CONCLUSTONS}

Le problème du miel de Guinée ne présente sur le plan technique aucune difficulté, il suffit de se convaincre de la nécessité de traiter à partir de la brèche pour éviter la souillure et la fraude.

Les procédés d'extraction doivent être adaptés à la brèche.

La commercialisation a prouvé qu'un miel extrait proprement pouvait prétendre sur le marché métropolitain à un prix largement rémunérateur pour la colonie.

Ia création d'une apiculture fixiste, si primitive soit-elle, est subordonnée à l'éducation d'apiculteurs.

Lia ruche à cadres qui ne peut s'adresser qu'à une élite doit, dans l'immédiat, rester sur le plan expérimental.

En pays Foulah surtout, plus que le labourage des terres érodées, plus que le pâturage des pacages incendiès, l'apiculture doit occuper une place importante : par son rapport immédiat et par la rentabilité du reboisement naturel. 